\title{
Envolvimento acadêmico no ensino superior e características do estudante ${ }^{1}$
}

\author{
Camila Alves Fior ${ }^{2}$ \\ Elizabeth Mercuri \\ Universidade Estadual de Campinas, Campinas-SP, Brasil
}

\section{Resumo}

O envolvimento acadêmico é um preditor da aprendizagem, desenvolvimento e permanência do estudante no ensino superior e é influenciado por diversas variáveis. O objetivo deste estudo é analisar as diferenças no envolvimento acadêmico, levando-se em consideração variáveis pessoais dos estudantes. Os dados, coletados através da Escala de Envolvimento Acadêmico, com 1000 universitários matriculados em uma instituição confessional, foram analisados através de estatística multivariada. Destaca-se a existência de diferenças estatisticamente significantes entre o envolvimento acadêmico com as atividades obrigatórias e com as não obrigatórias em função de características pessoais dos estudantes. Reafirma-se o dinamismo do envolvimento e a importância de conhecer suas características para o planejamento institucional.

Palavras-chave: Características do estudante, envolvimento, ensino superior, estudantes universitários

\section{Abstract: Academic involvement in higher education and students' characteristics}

Academic involvement is a predictor of learning, development and permanence of higher-education students and is influenced by several variables. This study aims to analyze the differences in academic involvement, considering students' personal variables. The data, collected using the Academic Involvement Scale with 1000 college students enrolled in a confessional institution, was analyzed using multivariate statistics. It is important to point out the existence of statistically significant differences between the academic involvement with mandatory and nonmandatory activities considering the students' personal variables. The results reinforce the dynamic aspect of involvement, and the importance of knowing its characteristics for institutional planning.

Keywords: Student characteristics, involvement, higher education, college students

\section{Resumen: Involucramiento académico en la educación superior y características de los estudiantes}

El involucramiento académico está determinado por diversas variables y es un predictor del aprendizaje, desarrollo y permanencia del estudiante en la educación superior. El objetivo de este estudio es analizar las diferencias con relación al involucramiento académico, teniendo en cuenta variables personales del estudiante. Los datos recolectados a través de la Escala de Involucramiento Académico con mil universitarios matriculados en una institución confesional fueron analizados por medio de la estadística multivariada. Se destaca la existencia de diferencias estadísticamente significativas entre el involucramiento académico con las actividades obligatorias y con las no obligatorias en función de características personales de los estudiantes. Se reafirma el dinamismo del involucramiento y la importancia de conocer sus características para la planificación institucional.

Palabras clave: Características del estudiante, involucramiento, educación superior, estudiantes universitarios

\footnotetext{
${ }^{1}$ As autoras agradecem à PUC-MG pelo auxílio financeiro através do Programa FIP n.5745.

${ }^{2}$ Endereço para correspondência: Faculdade de Educação da Unicamp - Departamento de Psicologia Educacional. Avenida Bertrand Russell, 801, Cidade Universitária Zeferino Vaz, 13083-865, Campinas, SP. E-mail: cafior@unicamp.br
} 
Desde o final da década de 60 do século XX, o ensino superior brasileiro vivenciou uma grande expansão, com aumento no número de instituições e de alunos matriculados. Concomitante à ampliação no acesso, um desafio enfrentado pela educação superior refere-se à qualidade da formação ofertada. Soares e Cunha (2017) consideram esta reflexão urgente na sociedade contemporânea, pautada pela incerteza e pela complexidade. Sobre a formação dos estudantes, a qualidade da educação superior relaciona-se à capacitação técnica e profissional e ao desenvolvimento de um conjunto de habilidades indispensáveis para a vida social, democrática e política (Bertolin, 2017).

As discussões sobre os objetivos da educação superior e sobre a qualidade do processo de formação não são recentes e não se restringem ao contexto nacional. Diversos países trabalham no aprimoramento dos indicadores que possam mensurar a qualidade da formação ofertada (McCormick, Kinzie, \& Gonyea, 2013; Souza, 2017). No Brasil, desde os anos finais da década de 1990, o Ministério da Educação investe na elaboração de sistemas de avaliação e, em 2004, instituiu o Sistema Nacional de Avaliação da Educação Superior (Sinaes). Trata-se de uma estrutura com foco na instituição, nos cursos e no desempenho dos estudantes. Martins e Ribeiro (2017) acrescentam que a ótica da regulação e avaliação atuais do sistema de educação superior nacional baseia-se na análise da instituição de ensino e dos resultados da aprendizagem do estudante. Há uma lacuna no que diz respeito à compreensão das interações do aluno com as instituições de ensino, com a escassez de indicadores que permitam mensurar a relação dos estudantes com os recursos e as experiências disponibilizados pela instituição.

$\mathrm{Na}$ literatura internacional, constata-se uma intensa produção científica com análises detalhadas das experiências que se associam a consequências educacionais desejáveis (Mayhew et al., 2016; Pascarella \& Terenzini, 2005). Esses estudos fundamentam-se na concepção proposta por Astin (1993) sobre o impacto da vivência universitária no estudante. Concebe-se que os estudantes ingressam no ensino superior com atributos e características pessoais, incluindo o background familiar e a história prévia de escolarização, que influenciam diretamente o processo de aprendizagem e desenvolvimento e que os levam a se envolver nas distintas experiências que são disponibilizadas pela instituição. A participação nessas atividades se associa a resultados, os quais incluem a aprendizagem, a permanência e o desenvolvimento, nas suas diversas expressões: afetivas, cognitivas e sociais. Neste sentido, as consequências educacionais desejáveis dependem do planejamento e da oferta de experiências distintas que se encontram sob responsabilidade das instituições e, também, do envolvimento dos estudantes nessas atividades. Kuh (2005) já destacava que depois de controladas as características de background, a participação do estudante nas diversas experiências é um fator chave para o sucesso acadêmico.

Historicamente, a concepção de envolvimento começa a ser construída a partir dos estudos de Tyler na década de 1930, com a menção ao tempo despendido em uma tarefa (Kuh, 2009). Posteriormente, Pace (1980) introduziu o conceito de qualidade do esforço e desenvolveu o College Student Experience Questionnaire (CSEQ) para mensurar o empenho do estudante e identificar as atividades que contribuem para aprendizagem e desenvolvimento pessoal (Kuh, 2009). Ao longo de quase cinco décadas de estudo com o instrumento afirma-se que quanto mais tempo e energia os estudantes devotam às tarefas, maiores são os benefícios (Chi, Liu, \& Bai, 2017). Ainda na década de 1980, Astin propôs o princípio do Envolvimento e o descreve como a quantidade e a qualidade de tempo e de esforço dedicados às diversas atividades acadêmicas (Astin, 1984).

Kuh é um dos autores com histórico de estudo do envolvimento acadêmico e que, no final da década de 1990, direciona seus trabalhos para um conjunto particular de experiências, inspirado na formulação de Chickering e Gamson (1987) sobre os sete princípios para uma boa prática educativa. $\mathrm{O}$ autor manifesta uma preocupação com as ações que são realizadas pela instituição para viabilizar o envolvimento dos estudantes nas atividades presentes no ensino superior (Kuh, 2009). A partir de tais estudos, Kuh introduz o conceito de engajamento e o caracteriza como apresentando duas dimensões. A primeira diz respeito ao tempo e ao esforço despendido pelo estudante para com um conjunto de atividades e que são associadas a consequências desejáveis. Uma segunda dimensão refere-se às práticas implementadas pela instituição para incentivar a participação dos alunos nestas experiências. O autor trabalhou na construção do National Survey of Student Engagement (NSSE), instrumento norte-americano amplamente utilizado com a finalidade de mensurar o impacto da experiência acadêmica, adaptado, inclusive, para outros países. (Kahu, 2013).

Constata-se, portanto, a existência de termos distintos: esforço, engajamento e envolvimento para nomearem o investimento de tempo e energia com atividades acadêmicas. Os autores concordam que a aprendizagem e o desenvolvimento dos estudantes são intimamente associados às ações e à participação ativa nas diversas tarefas presentes na vida acadêmica (Astin, 1984; Kuh et al., 1991; Pace, 1980).

Astin (1984) já apontava que o conceito de esforço tem muito em comum com a concepção de envolvimento. Para Kuh (2009) o engajamento está vinculado 
à participação exclusiva em experiências que já foram descritas na literatura como importantes para a formação, tais como aprendizagem ativa e colaborativa, interações professores-alunos, ambientes de suporte no campus. Concebe o engajamento, como já mencionado, composto por duas dimensões: o que o estudante faz e o que a instituição faz.

Wolf-Wendel, Ward e Kinzie (2009) explicitam que engajamento e envolvimento são conceitos distintos. Acrescentam que a concepção de envolvimento está centralizada nas ações que são realizadas pelos estudantes. Essa ideia não isenta a instituição de sua responsabilidade no planejamento das atividades, mas focaliza na prática do aluno. Por sua vez, a ênfase do engajamento, conforme proposição de Kuh (2009), está no desenvolvimento de contexto institucional que disponibiliza diversas experiências para o estudante. Wolf-Wendel et al. (2009) destacam que as pesquisas sobre engajamento focalizam a instituição, ao invés do estudante e, apesar de serem realizadas sob o olhar do universitário, especificamente às que utilizam o NSSE, incorporam as características das instituições que favoreçam a participação e analisam a efetividade das práticas institucionais. Por sua vez, sabe-se que essa não é a única perspectiva para a compreensão do engajamento, mas é bastante presente nas últimas décadas (Kahu, 2013).

Wolf-Wendel et al. (2009) consideram que o peso das evidências empíricas mantém a importância de ambas teorias: envolvimento e engajamento para a compreensão do sucesso acadêmico. Harper e Quaye (2009) acrescentam que a proposta de Astin é uma das mais citadas na literatura da educação superior. Sem desmerecer os componentes afetivos e cognitivos vinculados ao conceito de envolvimento, Astin (1984) enfatiza a importância das análises sobre os aspectos comportamentais da vivência do estudante, ou seja, o que faz ou como se comporta a fim de compreender a sua participação no ensino superior. Concebe que o envolvimento é composto por dimensões qualitativas e quantitativas: o que o aluno faz e o quanto faz. Além disso, trata-se de uma variável que não é estática, mas que varia no decorrer da trajetória acadêmica. $\mathrm{O}$ estudante manifesta graus distintos de envolvimento em experiências diferentes no decorrer de sua formação. Diante disso, o presente estudo opta por focalizar o envolvimento acadêmico como a dimensão a ser avaliada.

O envolvimento do estudante com as experiências acadêmicas é uma variável importante, altamente associada com o desempenho (Loes, Pascarella, \& Umbach, 2012), com a satisfação com a experiência acadêmica (Webber, Krylow, \& Zang, 2013), com a permanência (Shappie \& Debb, 2017), com o desenvolvimento intelectual (Chi et al., 2017) e com habilidades de liderança (Wu, 2011). Por sua vez, é importante que se conheçam as características das experiências com as quais o estudante se envolve, visto que os impactos anteriormente descritos dependem da natureza das experiências vivenciadas. Dentre os aspectos mais importantes apontados na literatura estão: quantidade de tempo despendido nos estudos, interação com os agentes de socialização (professores e colegas), participação em projetos de pesquisa e a utilização de recursos institucionais. (Astin, 1993; Kuh et al., 1991).

Kuh $(1993,1995)$, no contexto internacional, e Fior e Mercuri (2003) e Porto e Gonçalves (2017), através de estudos realizados no Brasil, apontam que o estudante se envolve em uma diversidade de experiências ao longo da graduação, incluindo as atividades obrigatórias e não obrigatórias. Nesses estudos, as atividades obrigatórias constituem-se em um conjunto de experiências que têm relação direta com o cumprimento das exigências de um curso superior. Sabe-se que o currículo universitário não se restringe à grade curricular ou ao conjunto de tarefas obrigatórias, mas incluem outras experiências, denominadas não obrigatórias (Fior \& Mercuri, 2003). Segundo as autoras, essas são atividades vivenciadas pelos estudantes; isoladamente, não são comuns aos alunos do mesmo curso e também não são imprescindíveis para a integralização da graduação do estudante, mas são experiências que se encontram sob responsabilidade da instituição.

Apesar de ainda existirem propostas de formação universitária que se centralizam nas experiências consideradas obrigatórias, a importância do envolvimento com as atividades não obrigatórias parece ser consenso na literatura (Kuh et al., 1991; Kuh, 1993, 1995; Fior \& Mercuri, 2003; Oliveira, Santos, \& Dias, 2016). Porém, de acordo com Fior e Mercuri (2009), não se deve desmerecer as tarefas obrigatórias, estando nelas o grande papel das ações educativas.

O envolvimento do estudante varia de acordo com as características das instituições, como também, do background e de atributos pessoais do aluno, entre outras variáveis que formam uma rede complexa de influências que devem ser levadas em consideração nos estudos que tenham como interesse o envolvimento dos estudantes (Kuh \& Vésper, 1997; Porto \& Gonçalves, 2017). Wu (2011), em pesquisa com estudantes de Taiwan sobre o impacto das distintas experiências presentes no ensino superior na capacidade de liderança, não encontrou diferenças de gênero no que diz respeito ao envolvimento dos estudantes. Todavia, Ro \& Knight (2016) encontram que homens e mulheres que cursam engenharia têm preferências distintas pelas experiências consideradas curriculares e que o impacto das mesmas também é 
diferente no que diz respeito aos interesses, autoeficácia e aprendizagem.

Pace (1980) ressaltou que o envolvimento dos estudantes aumenta com os anos de graduação, sendo a diferença estatisticamente significante. Isso sugere que a participação acadêmica dos estudantes é distinta entre calouros e concluintes. Outros estudos destacam que o papel das diversas experiências é diferente, levando-se em consideração variáveis pessoais como: residir no campus ou viajar diariamente, trabalhar durante a graduação, entre outros aspectos (Mayhem et al., 2016). Estudos apontam que os estudantes com nível socioeconômico mais baixo trabalham mais durante a graduação e têm menos tempo para o envolvimento com as atividades extracurriculares quando comparados aos estudantes que não trabalham (Astin, 1993; Nora \& Crisp, 2009).

Os resultados da pesquisa de Logan, Hughes e Logan (2016) indicam que os estudantes que trabalham mais do que 20 horas por semana têm rendimento acadêmico mais baixo. Uma vez que o envolvimento é um importante preditor do desempenho, é provável que o estudante que trabalhe tenha dificuldade em se envolver com as distintas experiências que poderiam associar-se a melhores resultados acadêmicos. Os resultados sugerem, ainda, que os impactos negativos do número de horas trabalhadas sobre a vivência do estudante estão presentes tanto para os ingressantes como para os concluintes. No contexto nacional, o estudante-trabalhador, tradicionalmente, tende a se matricular em cursos que funcionam no período noturno (Vargas \& Paula, 2013). Portanto, infere-se que o aluno que frequenta a instituição no período noturno, pela necessidade de conciliar estudo e trabalho, enfatiza na sua formação o envolvimento com as experiências obrigatórias.

Apesar das instituições exercerem um papel no envolvimento do estudante, através do investimento de recursos e organização dos currículos e dos contextos de aprendizagem e dos serviços de auxílio sabe-se, como visto, que características pessoais do estudante também influenciam o envolvimento. Conhecer essa atuação pode contribuir para a formatação de propostas que ampliem a participação dos estudantes nas diversas atividades associadas à sua aprendizagem e ao seu desenvolvimento.

Diante disso, o objetivo do presente estudo é analisar as diferenças no envolvimento acadêmico, especificamente com as atividades obrigatórias e não obrigatórias, levando-se em consideração variáveis pessoais dos estudantes, que incluem: gênero, idade, curso, momento do curso (ingressante ou concluinte), turno do curso (diurno ou noturno), exercício de atividade remunerada e horas semanais de trabalho.

\section{Método}

\section{Participantes}

Este estudo foi realizado com uma amostra não aleatória, por conveniência, de 1000 estudantes matriculados em uma das maiores universidades confessionais do país, localizada na região Sudeste. Na seleção dos participantes buscou-se aqueles matriculados nos períodos iniciais e finais do curso. Foram considerados alunos ingressantes os inscritos no primeiro ou no segundo semestre de curso e concluintes aqueles matriculados nos dois últimos semestres. A Tabela 1 apresenta detalhamento sobre as características da amostra pesquisada.

Ainda sobre a amostra, a média de idade dos participantes foi de 23 anos, com desvio padrão de 5,7. Os mesmos estavam matriculados nos seguintes cursos: Administração (18\%), Arquitetura (8,4\%), Ciências da Computação (10,5\%), Direito (29,2\%), Medicina Veterinária $(8,8 \%)$, Psicologia $(13,8 \%)$ e Engenharia Elétrica (11,3\%). A maioria dos estudantes não trabalhava $(53,2 \%)$, e dentre os que exerciam atividade remunerada, a média era de 16 horas semanais.

Tabela 1

Características sociodemográficas dos participantes da pesquisa

\begin{tabular}{|c|c|c|c|c|c|}
\hline \multirow[b]{2}{*}{ Momento do Curso } & \multirow[b]{2}{*}{$\begin{array}{l}\text { Total } \\
\text { N (\%) }\end{array}$} & \multicolumn{2}{|c|}{ Turno de Funcionamento do Curso } & \multicolumn{2}{|c|}{ Gênero } \\
\hline & & $\begin{array}{l}\text { Diurno } \\
\text { N (\%) }\end{array}$ & $\begin{array}{c}\text { Noturno } \\
\text { N (\%) }\end{array}$ & $\begin{array}{l}\text { Masculino } \\
\text { N (\%) }\end{array}$ & $\begin{array}{l}\text { Feminino } \\
\mathrm{N}(\%)\end{array}$ \\
\hline Ingressante & $583(58,3 \%)$ & $248(24,8 \%)$ & $335(33,5 \%)$ & $295(29,5 \%)$ & $288(28,8 \%)$ \\
\hline Concluinte & $417(41,7 \%)$ & $246(24,6 \%)$ & $171(17,1 \%)$ & $199(19,9 \%)$ & $218(21,8 \%)$ \\
\hline TOTAL & $1000(100 \%)$ & $494(49,4 \%)$ & $506(50,6 \%)$ & $494(49,4 \%)$ & $506(50,6 \%)$ \\
\hline
\end{tabular}




\section{Instrumentos}

Para a coleta de dados utilizou-se a Escala de Envolvimento Acadêmico, desenvolvida por Fior, Mercuri e Silva (2013), especificamente para a análise do envolvimento acadêmico de estudantes do ensino superior.

A escala é composta por 23 afirmações sobre o envolvimento acadêmico, construídos em formato do tipo Likert, que devem ser respondidas através de uma escala de frequência de cinco pontos. As afirmações descrevem um conjunto de atividades com as quais os alunos se envolvem e as opções de respostas variavam do $1=$ "nada/ nunca" até 5 = "muito/sempre".

Os itens que compõem a escala agrupam-se em dois fatores. O primeiro é denominado por "envolvimento com as atividades obrigatórias" e é formado por 14 itens, como, por exemplo, "Estudo para as avaliações". O segundo fator denomina-se por "envolvimento com as atividades não obrigatórias", compõe-se por nove afirmações, ilustradas por "Frequento atividades de monitoria ou tutoria". Além disso, dois itens referentes ao domínio "envolvimento com as atividades obrigatórias" foram construídos no sentido negativo, de modo que uma frequência mais elevada indica baixo envolvimento acadêmico e fez-se a inversão dos escores no momento de tabulação dos dados.

Com relação às propriedades psicométricas, o alpha de Cronbach da escala na sua totalidade é 0,853 , o do fator "envolvimento com atividades obrigatórias" 0,85 e do segundo fator, "envolvimento com as atividades não obrigatórias", 0,73 . Tais valores são indicativos de uma boa consistência interna do instrumento.

\section{Procedimentos}

A aplicação da Escala de Envolvimento Acadêmico ocorreu tanto de maneira individual como coletiva e a duração média da coleta foi de 20 minutos. Os estudantes foram contatados em diferentes ambientes do convívio universitário, como salas de aula, bibliotecas, cantinas, Diretórios Acadêmicos, sendo a recolha dos dados realizada na própria instituição. A pesquisa foi submetida ao Comitê de Ética em Pesquisa com seres humanos, com aprovação sob protocolo CAAE - 01850.146.000-08.

A análise dos dados foi realizada através do pacote estatístico Statistical Analysis System (SAS System for Windows), versão 8.02. Os dados foram submetidos a análises estatísticas descritivas e inferenciais. Foram calculadas a média e o desvio-padrão da escala na sua totalidade e em cada uma das subescalas do instrumento. Optou-se pelo parâmetro listwise para o trabalho com os missings. Em continuidade às análises, através do teste de normalidade Kolmogorov-Smirnov constatou-se que alguns dados não eram normais e, por isso, optou-se pela transformação dos mesmos em postos ou ranks a fim de normalizá-los. Também foi analisada a homocedasticidade dos dados, a qual mostrou-se adequada para a realização dos testes paramétricos.

As análises estatísticas realizadas envolveram inicialmente os escores totais da Escala de Envolvimento Acadêmico, o que inclui as atividades obrigatórias e as não obrigatórias, considerando as variáveis pessoais dos estudantes: gênero, idade, curso, momento do curso, turno do curso, exercício de atividade remunerada e horas trabalhadas. Este estudo inicial foi realizado através da análise de variância multivariada (MANOVA). Ainda relacionadas às características dos estudantes, foram realizadas análises de variância (ANOVA) para cada um dos fatores da Escala de Envolvimento Acadêmico, a fim de serem identificadas as características pessoais do estudante que influenciam a variação no envolvimento. $\mathrm{O}$ nível de significância adotado para os testes estatísticos foi de $5 \%$, ou seja, $p \leq 0.05$.

\section{Resultados e Discussão}

Considerando a totalidade dos participantes da pesquisa, contatou-se que a média geral de envolvimento, no decorrer da trajetória acadêmica, foi $M=3,26$; $D P=$ 0,52 , o que indica um envolvimento moderado com o conjunto das atividades acadêmicas. Um aprofundamento da compreensão deste valor é possível a partir da análise das médias de envolvimento com as atividades obrigatórias $(M=3,71 ; D P=0,59)$ e das não obrigatórias $(M=2,58$; $D P=0,66)$. Verifica-se que a amostra do presente estudo se envolve mais com as atividades obrigatórias do que com as não obrigatórias, sendo que estas últimas ocorrem com frequência inferior ao ponto médio da escala, enquanto que para envolvimento com as atividades obrigatórias, a média de frequência é superior ao ponto médio.

Com relação aos resultados do presente estudo, procedeu-se a uma etapa de análise na qual buscou-se compreender a significância estatística das diferenças entre os escores totais de Envolvimento Acadêmico e características pessoais dos estudantes. A Tabela 2 apresenta os resultados da análise multivariada (Manova - Multivariate Analysis of Variance) os quais indicam que as variáveis momento do curso, gênero, idade, curso, exercício de atividade remunerada, número de horas trabalhadas explicam, significativamente, a variabilidade observada nos escores totais do envolvimento acadêmico com as atividades obrigatórias e com as não obrigatórias. Por outro lado, uma única variável, turno do curso no qual o aluno está matriculado no ensino superior, que poderia ser diurno ou noturno, não explicou a 
variabilidade nos escores de envolvimento acadêmico com as atividades obrigatórias e não obrigatórias.

A partir da constatação de que variáveis pessoais do estudante contribuem para a explicação na variabilidade do envolvimento acadêmico, procedeu-se a uma nova etapa das análises, na qual buscou-se discriminar os efeitos das variáveis do estudante sobre cada um dos dois escores que compõem o envolvimento acadêmico, através da Anova (Analysis of Variance). Dos resultados apresentados na Tabela 3 destaca-se que tanto o envolvimento acadêmico com as atividades obrigatórias, quanto aquele com atividades não obrigatórias, mostram-se associados com variáveis pessoais dos estudantes. Porém, enquanto os escores de envolvimento com atividades obrigatórias associam-se significativamente com momento do curso, curso realizado, gênero, idade, os escores de envolvimento com atividades não obrigatórias relacionam-se significativamente com momento do curso, curso realizado, e com atividade remunerada e horas de trabalho. Portanto, o momento do curso, que se refere ao fato de ser ingressante ou concluinte e o curso realizado constituem-se em variáveis pessoais que relacionam-se com ambas formas de envolvimento. Por outro lado, gênero e idade dos estudantes mostram-se associados exclusivamente com envolvimento com atividades obrigatórias e as variáveis exercício ou não de atividade remunerada e horas de trabalho relacionadas apenas com o envolvimento com atividades não obrigatórias. Afirma-se que as variáveis pessoais dos estudantes associam-se significativamente com o envolvimento acadêmico e apresentam especificidades, com diferentes características de associação quando se considera a natureza das atividades: obrigatórias e não obrigatórias.

Tabela 2

Diferenças no envolvimento acadêmico (total), considerando variáveis pessoais dos estudantes

\begin{tabular}{lcl}
\hline \multicolumn{1}{c}{ Variáveis Independentes } & Categorias & \multicolumn{1}{c}{ Manova } \\
\hline Momento do curso & Ingressante/Concluinte & $\Lambda=0,864 ; \mathrm{F}=73,97 ; p<0,001^{* *}$ \\
Gênero & $\mathrm{M} / \mathrm{F}$ & $\Lambda=0.967 ; \mathrm{F}=15,87 ; p<0,001^{* *}$ \\
Curso & 7 Cursos & $\Lambda=0,866 ; \mathrm{F}=11,66 ; p<0,001^{* *}$ \\
Turno & Diurno/Noturno & $\Lambda=0.998 ; \mathrm{F}=0,93 ; p=0,396$ \\
Atividade remunerada & Sim/Não & $\Lambda=0,991 ; \mathrm{F}=4,48 ; p=0,012^{*}$ \\
Horas trabalho & Variável contínua (horas) & $\Lambda=0,994 ; \mathrm{F}=3,05 ; p=0,048^{*}$ \\
Idade & Variável contínua (anos) & $\Lambda=0,983 ; \mathrm{F}=8,01 ; p<0,001^{* *}$ \\
\hline
\end{tabular}

$\Lambda-$ Wilks' Lambda; ${ }^{* *} p \leq 0,001 ; * p \leq 0,05$

É possível observar na Tabela 3 que com relação à participação nas atividades obrigatórias, os alunos de início de curso se envolvem mais com essas experiências do que os matriculados no final do curso. As mulheres se envolvem mais com as mesmas quando comparadas aos homens. E o envolvimento é maior para os alunos com maior idade. Quanto ao curso frequentado, maior envolvimento com atividades obrigatórias para aqueles matriculados nos cursos de Direito e Psicologia quando equiparados aos estudantes de Administração, Arquitetura, Ciências da Computação, Medicina Veterinária e Engenharia Elétrica.

Pela análise das diferenças no envolvimento dos alunos com as atividades não obrigatórias observa-se que os alunos concluintes se envolvem mais com as experiências não obrigatórias quando comparados aos de início de curso. Os alunos dos cursos de Medicina Veterinária, Psicologia e Arquitetura participam mais das práticas não obrigatórias do que seus colegas matriculados nos cursos de Administração, Ciências da Computação, Engenharia Elétrica e Direito. E os estudantes que não trabalham indicam maior envolvimento nas atividades não obrigatórias, quando comparados aos pares que exercem atividades remuneradas, sendo que aqueles com menos horas de trabalho participam mais nas propostas não obrigatórias.

Em síntese, verifica-se a existência de diferenças estatisticamente significantes entre o envolvimento dos estudantes com as atividades obrigatórias e não obrigatórias, quando se analisam características pessoais dos mesmos, sendo que duas se destacaram: momento do curso e o curso no qual estão matriculados. Os ingressantes relataram envolvimento maior com as atividades obrigatórias e os concluintes com as não obrigatórias. Com relação aos cursos, merece destaque o fato de os estudantes de Psicologia vivenciarem uma participação mais intensa em ambas as experiências, os alunos de Direito se comprometem mais nas atividades obrigatórias e os vinculados à Medicina Veterinária e Arquitetura se envolvem mais com as experiências não obrigatórias.

Neste estudo, a maior participação nas experiências obrigatórias coincide com os dados de Porto e Gonçalves 
(2017), em pesquisa realizada também com estudantes brasileiros. Segundo as autoras, as atividades obrigatórias são exigências para a conclusão do curso e imprescindíveis para a formação profissional. Por sua vez, permanecem questionamentos sobre as propostas de formação que se centralizam num conjunto extenso de experiências de natureza obrigatória, que podem limitar a vivência aos cenários restritos a essas atividades. Há décadas, o excesso de horas de aulas já se destacava como justificativa apontada por estudantes e por professores para explicar a inexistência de tempo suficiente para a realização das atividades de estudo (Mercuri, 1994).

Tabela 3

Diferenças no envolvimento com atividades obrigatórias (EAO) e não obrigatórias (EANO), considerando características dos estudantes (ANOVA)

\begin{tabular}{|c|c|c|c|c|}
\hline $\begin{array}{l}\text { Variáveis pessoais } \\
\text { dos estudantes }\end{array}$ & $\begin{array}{l}\text { Médias } \\
\text { EAO }\end{array}$ & $\begin{array}{c}\text { Envolvimento Atividades } \\
\text { Obrigatórias }\end{array}$ & $\begin{array}{l}\text { Médias } \\
\text { EANO }\end{array}$ & $\begin{array}{c}\text { Envolvimento Atividades } \\
\text { não obrigatórias }\end{array}$ \\
\hline Efeito Geral & & $\begin{array}{l}\mathrm{F}=15,63 \\
p<0.001^{* *}\end{array}$ & & $\begin{array}{l}\mathrm{F}=11,72 \\
p<0,001 * *\end{array}$ \\
\hline Ingressante & 3,84 & $\mathrm{~F}=69,74$ & 2,51 & $\mathrm{~F}=15,20$ \\
\hline Concluinte & 3,53 & $p<0,001 * *$ & 2,68 & $\mathrm{p}<0,001 * *$ \\
\hline Masculino & 3,60 & $\mathrm{~F}=22,43$ & 2,55 & $F=0,23$ \\
\hline Feminino & 3,81 & $p<0,001 * *$ & 2,62 & $p=0,633$ \\
\hline Administração & 3,47 & & 2,38 & \\
\hline Arquitetura & 3,59 & & 2,76 & \\
\hline C.Computação & 3,65 & & 2,38 & \\
\hline Direito & 3,88 & $\begin{array}{l}\mathrm{F}=8,06 \\
2\end{array}$ & 2,44 & $\begin{array}{l}\mathrm{F}=11,05 ; \\
n<0\end{array}$ \\
\hline Med. Vet. & 3,77 & & 3,07 & \\
\hline Psicologia & 3,86 & & 2,86 & \\
\hline Eng.Elétrica & 3,57 & & 2,57 & \\
\hline Diurno & 3,77 & $F=0,33$ & 2,73 & $F=0,67$ \\
\hline Noturno & 3,65 & $p=0,565$ & 2,44 & $p=0,412$ \\
\hline Trabalha & 3,64 & $F=0,16$ & 2,53 & $F=5,93$ \\
\hline Não Trabalha & 3,77 & $p=0,692$ & 2,63 & $p=0,015^{*}$ \\
\hline $\begin{array}{l}\text { Horas trabalho } \\
\text { (var. contínua) }\end{array}$ & & $\begin{array}{l}\mathrm{F}=0,01 \\
p=0,923\end{array}$ & $r=-0,078$ & $\begin{array}{c}\mathrm{F}=4,55 \\
p=0,033^{*}\end{array}$ \\
\hline $\begin{array}{l}\text { Idade } \\
\text { (var. contínua) }\end{array}$ & $r=0,081$ & $\begin{array}{c}\mathrm{F}=5,73 \\
p=0,017^{*}\end{array}$ & & $\begin{array}{l}\mathrm{F}=2,93 \\
p=0,087\end{array}$ \\
\hline
\end{tabular}

** $p \leq 0,001 ; * p \leq 0,05 ; r$-Coeficiente de correlação parcializado de Spearman

A compreensão dos motivos pelos quais os participantes do presente estudo se envolvem mais com as atividades obrigatórias deve considerar os aspectos pessoais do aluno, as características do funcionamento institucional e aspectos culturais. Wu (2011) destaca que, tradicionalmente, a cultura oriental tende a valorizar a participação nas atividades obrigatórias, em detrimento das não obrigatórias, com receio de que esta última possa impactar negativamente a formação do aluno, devido ao acúmulo de atividades a serem desempenhadas. A educação básica nacional, em sua maioria, centraliza-se nas experiências obrigatórias e a ausência de ações intencionais que discutam com o aluno em formação o papel de práticas distintas no seu sucesso acadêmico poderá levá-los a reproduzirem as experiências de formação vividas na sua história prévia de escolarização e centralizadas nas atividades obrigatórias. Coulon (2016) complementa tais reflexões ao descrever que, muitas vezes, os estudantes e suas famílias não 
percebem as rupturas existentes entre o ensino médio e superior, especificamente no que diz respeito à apropriação dos saberes, e manifestam uma continuidade nas práticas com ênfase nas experiências de sala de aula.

Apesar da importância das atividades obrigatórias na formação do estudante, Oliveira et al. (2016) acrescentam que tais experiências podem não ser suficientes para a promoção de todas as habilidades necessárias para a atuação profissional. A presença das atividades não obrigatórias tem uma importância ímpar na trajetória acadêmica do estudante e para a sua formação (Kuh, 1995; Fior \& Mercuri, 2009; Oliveira et al., 2016). No presente estudo, considerando que todos os estudantes estavam matriculados na mesma instituição, a diferença de envolvimento acadêmico, quando se leva em consideração o curso, pode ser reflexo dos projetos políticos-pedagógicos, que são específicos a cada curso. Sabe-se que há propostas de formação que incorporaram os princípios da flexibilização curricular e trazem, como complementares às experiências obrigatórias, vivências com características extras e optativas aos alunos (Fior \& Mercuri, 2009). Reforçam esta ideia de diferenças intrainstituição os estudos de $\mathrm{Hu}$ e McCormick (2012) que apontam que a participação dos estudantes nas distintas experiências varia mais entre os matriculados em uma mesma instituição, do que entre instituições distintas. E o trabalho de Porto e Gonçalves (2017), com estudantes brasileiros, que também identificaram frequência de participações distintas no envolvimento com as atividades obrigatórias e não obrigatórias, entre estudantes matriculados em cursos distintos. A existência de diferentes padrões de envolvimento entre os cursos resulta em consequências também diversas para os estudantes e reafirma a responsabilidade da instituição no planejamento das experiências ofertadas aos alunos.

Com relação às diferenças encontradas no envolvimento acadêmico em relação ao momento do curso, os dados são semelhantes aos relatados por Porto e Gonçalves (2017) com maiores escores de envolvimento com atividades obrigatórias entre estudantes ingressantes e uma participação maior dos concluintes nas experiências não obrigatórias. A participação em atividades não obrigatórias exige do estudante um conhecimento amplo da estrutura do ensino superior, das experiências que são disponibilizadas e uma maior integração ao sistema universitário (Oliveira et al., 2016). Na transição do ensino médio para o superior, o estudante ingressante depara-se com desafios que incluem a construção de uma nova forma de gestão da vida pessoal, a reorganização da rede social, o desenvolvimento de habilidades acadêmicas, e o conhecimento sobre a estrutura e a rotina da instituição superior (Coulon, 2016). É provável que o aluno ingressante, ainda em transição para o ensino superior, encontre na participação em atividades obrigatórias um contexto importante para a sua integração.

Sobre os alunos concluintes, os mesmos participam com maior intensidade nas experiências que excedem os limites da sala de aula e das exigências obrigatórias de um curso quando comparados aos ingressantes. Esse maior envolvimento pode ser em parte reflexo das práticas institucionais que, muitas vezes, oportunizam tais vivências apenas a alunos matriculados em períodos mais avançados (Oliveira et al., 2016). As autoras acrescentam que a participação nas atividades não obrigatórias exige do aluno uma postura mais autônoma, ao perceberem a importância de imprimirem diferenciais na sua formação e assumirem um papel mais ativo na sua aprendizagem, sendo a autonomia uma habilidade desenvolvida no decorrer da vivência no ensino superior (Chickering \& Reisser, 1993).

No que diz respeito à variável gênero, observou-se que as mulheres se envolvem mais nas atividades obrigatórias, quando comparadas aos homens, não havendo diferenças quanto ao envolvimento nas atividades não obrigatórias. Tais resultados são coerentes com os achados de Webber, Krylow e Zhang (2013) que afirmam que as mulheres se dedicam mais às atividades vinculadas à sala de aula e com outras tarefas relacionadas às atividades obrigatórias, o que resulta na ampliação do rendimento acadêmico. A compreensão dos motivos pelos quais há diferenças entre homens e mulheres no envolvimento acadêmico passa por análises sobre a construção do gênero na sociedade contemporânea.

Sobre o envolvimento de estudantes de diferentes idades, verificou-se, em relação às atividades de natureza obrigatória, que os estudantes mais velhos quando comparados aos mais jovens, se envolvem mais fortemente nestas atividades. Tais resultados dialogam com os estudos de McCormick, Kinzie e Gonyea (2013), os quais encontram que os estudantes mais velhos dedicam-se com mais afinco às experiências acadêmicas. Colaboram com essa reflexão as colocações de Quintas et al. (2014), ao acrescentarem que para tais estudantes a decisão de retomar os estudos e ingressar no ensino superior exige um amplo planejamento, a fim de conciliarem, muitas vezes, questões profissionais e familiares com o ensino superior. Ao mesmo tempo, trata-se de uma decisão bastante ponderada e vista por estes alunos como uma oportunidade ímpar para concretizarem um projeto que ficou inacabado em algum momento de sua vida. No contexto de sala de aula, os autores pontuam como positiva a percepção que os estudantes mais velhos têm sobre a possibilidade de incorporarem, no seu processo de formação, os saberes práticos. 
Com relação ao envolvimento com as atividades acadêmicas, sejam elas obrigatórias ou não obrigatórias, Kuh et al.(1991) destacaram que o envolvimento pressupõe um outro elemento: tempo despendido na atividade. Esta afirmativa auxilia na compreensão de que, neste estudo, os alunos que não exercem atividade remunerada têm uma participação maior nas atividades não obrigatórias, quando comparados aos demais que trabalham. Ainda sobre questões vinculadas ao trabalho, aqueles com menos horas de trabalho se envolvem mais nas atividades não obrigatórias. Portanto, a variação no envolvimento dos estudantes pode relacionar-se à disponibilidade de tempo que possuem para participar nas diversas experiências, sendo que o estudante trabalhador tem limitações temporais para sua vivência acadêmica, restringindo-se e igualando-se aos estudantes que não exercem atividades remunerados em relação ao envolvimento em atividades obrigatórias.

De acordo com Kuh et al. (1991), a participação nas distintas experiências acadêmicas proporciona ricas oportunidades de aprendizado e desenvolvimento para o estudante. Os autores pontuam que universitários que se envolvem com as atividades não obrigatórias tendem a estar mais satisfeitos com sua aprendizagem e sentem-se mais participativos na vida no campus.

Por sua vez, neste estudo, a pouca participação dos calouros com as experiências não obrigatórias merece atenção dos gestores educacionais. Astin (1993) e McCormick, Kinzie e Gonyea (2013) destacam que o envolvimento nas diversas atividades é associado à permanência do aluno, sugerindo que uma maior participação dos alunos ingressantes poderia reduzir a evasão neste nível de ensino.

Sobre o impacto do envolvimento acadêmico, Bowman e Trolian (2017) questionam a linearidade das relações entre envolvimento, aprendizagem e desenvolvimento. Para os autores, a relação entre tais variáveis é curvilínea, ou seja, uma baixa participação é ruim para a formação do estudante, porém, um alto envolvimento, principalmente com as atividades não obrigatórias, pode comprometer o rendimento acadêmico dos alunos. Isto porque o envolvimento pressupõe a variável tempo, e o aluno que despende tempo com as atividades não obrigatórias pode ter restrições temporais para participar das atividades obrigatórias.

\section{Considerações Finais}

A partir do objetivo de investigar as diferenças no envolvimento acadêmico tendo como parâmetro os atributos pessoais dos estudantes, os resultados obtidos nesse estudo confirmam o caráter dinâmico do envolvimento e destacam que as variáveis pessoais influenciam a participação dos estudantes nas diversas experiências presentes no ensino superior. Mayhew et al. (2016), através de uma extensa revisão de literatura sobre trabalhos com o estudante universitário, já alertavam que o impacto das vivências no ensino superior sobre o sucesso acadêmico é condicionado por variáveis, inclusive, as pessoais do estudante.

Neste estudo, constatou-se a existência de diferenças estatisticamente significantes no envolvimento acadêmico com as atividades obrigatórias e não obrigatórias quando se leva em consideração variáveis pessoais dos estudantes. Conhecer tais diferenças é imprescindível para desenvolvimento de programas e ações institucionais que ampliem o envolvimento acadêmico do estudante.

Além disso, uma vez que as instituições têm uma grande influência sobre o envolvimento do estudante, novos estudos deveriam incorporar particularidades do contexto e da cultura institucional, a fim de verificar o papel das características institucionais sobre o empenho dos alunos. Isso porque as universidades têm uma contribuição ímpar no envolvimento acadêmico dos seus alunos, seja através da formatação de projetos pedagógicos e currículos, ou do oferecimento de uma estrutura de suporte,

Dos limites do presente trabalho, destacam-se questões metodológicas relacionadas ao instrumento de coleta de dados. Sugere-se a urgência no aprimoramento de novos recursos de coleta de dados, principalmente que sejam capazes de incorporar a presença de experiências que possibilitem ao estudante a oportunidade de testar e aplicar os conteúdos aprendidos, através de resolução de problema e pelo uso de metodologias mais ativas envolvendo o processo de aprendizado (McCormick et al., 2013). Isto porque, segundo os autores, o que os estudantes aprendem é afetado pela forma como aprendem. Novos instrumentos deveriam olhar também para o papel da instituição e verificar como a mesma e os seus docentes estruturam as oportunidades de aprendizagem e fornecem feedback aos estudantes (McCormick et al., 2013). Também, a centralidade do instrumento na dimensão comportamental sinaliza limites na compreensão do envolvimento, visto que crenças e atitudes são antecedentes do comportamento e outros trabalhos deveriam incorporar as dimensões autorregulatórias do processo de envolvimento acadêmico, envolvendo aspectos cognitivos e afetivos (Kahu, 2013).

Num contexto no qual as instituições de ensino superior se abrem para grupos distintos, são imprescindíveis o planejamento e o suporte para o envolvimento acadêmico de grupos que tradicionalmente estiveram excluídos deste 
sistema de ensino (Shappie \& Debb, 2017). Kuh (2009) já afirmava que as pesquisas empíricas indicam que todos os estudantes se beneficiam das boas práticas educativas. Mas Cruce, Wolniak, Seifer e Pascarella (2006) acrescentam que o envolvimento acadêmico parece ter um efeito compensatório nas notas e permanência para estudantes que mais necessitam de suporte na transição do ensino médio para o ensino superior, devido ao fato de não estarem academicamente preparados ao ingressarem na universidade. Novos estudos nacionais sobre o envolvimento do estudante são necessários, visto que os maiores impactos das experiências universitárias são condicionais, significando que alguns estudantes se beneficiam mais do que outros de certas atividades (Mayhew et al., 2016).

Uma vez que as atividades desenvolvidas pelos estudantes durante o período de graduação têm um grande impacto em termos da aprendizagem, desenvolvimento e permanência dos estudantes, é urgente o direcionamento do olhar para o cotidiano da formação. Além disso, sugere-se um cuidado no planejamento das experiências que são disponibilizadas aos estudantes, a fim de favorecerem o sucesso acadêmico no ensino superior.

\section{Referências}

Astin, A.W. (1984). Student involvement: a developmental theory for higher education. Journal of College Student Personnel, 25(4), 297-308.

Astin, A.W. (1993). What matters in college? Four critical years revisited. San Francisco: Jossey-Bass Publisher.

Bertolin, J. (2017). A formação integral na educação superior e o desenvolvimento dos países. Cadernos de Pesquisa, $47(165), 848-871$.

Bowman, N., \& Trolian, T. (2017). Is More Always Better? The Curvilinear Relationships between College Student Experiences and Outcomes. Innovative Higher Education, 42(5/6), 477-489.

Chi, X., Liu J.,\& Bai Y. (2017). College environment, student involvement, and intellectual development: evidence in China. Higher Education. 74, 81-99.

Chickering, A.W.; \& Reisser, L. (1993). Education and identity. (2a ed). San Francisco: Jossey-Bass Publisher.

Chickering, A.W., \& Gamson, Z.F. (1987). Development and Adaptations of the Seven Principles for Good Practice in Undergraduate Education. AAHE Bulletin, 37(9), 3-7.

Coulon, Alain. (2017). O ofício de estudante: a entrada na vida universitária. Educação e Pesquisa, 43(4), 1239-1250.

Cruce, T., Wolniak, G. C., Seifert, T. A., \& Pascarella, E. T. (2006). Impacts of good practices on cognitive development, learning orientations, and graduate degree plans during the first year of college. Journal of College Student Development, 47(4), 365-383.

Fior, C. A., \& Mercuri, E. (2003). Formação universitária: o impacto das atividades não obrigatórias nas mudanças pessoais dos estudantes. In Mercuri, E. \& Polydoro, S.A.J. (Orgs.). Estudante universitário: características e experiências de formação. Taubaté: Editora Cabral,129-153.

Fior, C. A., \& Mercuri, E. (2009). Formação universitária e flexibilidade curricular: importância das atividades obrigatórias e não obrigatórias. Psicologia da Educação, 29(1), 191-215.

Fior, C. A., Mercuri, E., \& Silva, D. da. (2013). Evidências de validade da Escala de Envolvimento Acadêmico para universitários. Avaliação Psicológica, 12(1), 81-89.

Harper, S.R., \& Quaye, S.J. (2015). Student Engagement in Higher Education: Theoretical Perspectives and Practical Approaches for Diverse Populations. 2.ed. New York: Routledge.

Hu, S., \& McCormick, A. C. (2012). An engagement-based student typology and its relationship to college outcomes. Research in Higher Education, 53(7), 738-754.

Kahu, E.R. (2013) Framing student engagement in higher education. Studies in Higher Education, 38(5), 758-773

Kuh, G.D. (1993). In their own words: what students learn outside the classroom. American Educational Research Journal, 30(2), 277-304.

Kuh, G..D. (1995). The other curriculum: out-of-class experiences associated with student learning and personal development. Journal of Higher Education, 66(2), 123-155.

Kuh, G. (2005). Student engagement in the first year of college. In Upcraft, M.L, Gardner, J.N. \& Barefoot, B.O. Challenging and supporting the first-year student: A handbook for improving the first year of college, New Jersey: Jossey-Bass, 86-107.

Kuh, G. (2009). What student affairs professionals need to know about student engagement. Journal of College Student Development, 50(6), 683-706.

Kuh, G.D.; Schuh, J.H.; Whitt, E.J., Andreas, R.E., Lyons, J.W., Strange, C.C., Krehbiel, L.E., \& MacKay, K.A. (1991). Involving colleges: successful approaches to fostering student learning and development outside the classroom. San Francisco: Jossey-Bass Publisher. 
Kuh, G.D., \& Vesper, N. (1997). A comparasion of student experiences with good practices in undergraduate education between 1990 and 1994. The Review of Higher Education, 21(1), 43-61.

Loes, C., Pascarella, E., \& Umbach, P. (2012). Effects of diversity experiences on critical thinking skills: Who benefits? Journal of Higher Education, 83(1), 1-25.

Logan, J., Hughes, T., \& Logan, B. (2016). Overworked? An observation of the relationship between student employment and academic performance. Journal of College Student Retention: Research, Theory and Practice, 18, 250-262.

Martins, L. M. de, \& Ribeiro, J. L. D. (2017). Engajamento do estudante no ensino superior como indicador de avaliação. Avaliação: Revista da Avaliação da Educação Superior , 22(1), 223-247.

Mayhew, M. J., Rockenbach, A. N., Bowman, N. A., Seifert, T. A., Wolniak, G. C., Pascarella, E. T., \& Terenzini, P. Y. (2016). How college affects students: 21 st century evidence that higher education works (vol. 3). San Francisco, CA: Jossey-Bass.

McCormick, A.C., Kinzie, J., \& Gonyea, R.M. (2013). Student Engagement: Bridging Research and Practice to Improve the Quality of Undergraduate Education. In: Paulsen, M.B., Ed., Higher Education: Handbook of Theory and Research, 28. Springer Netherlands, 47-92.

Mercuri, E. (1994). Condições para o estudo, segundo depoimentos de alunos e professores de cursos de graduação. Pro-posições, 5(1), 61-71.

Nora, A., \& Crisp, G. (2009). Hispanics and Higher Education: An Overview of Research, Theory, and Practice. In J.C.Smart (Ed.) Handbook of Theory and Research in Higher Education (pp. 317-353). Neu-Isenberg, Germany: Springer Medizin Press.

Oliveira, C. T. de, Santos, A. S. dos, \& Dias, A. C. G.(2016). Percepções de Estudantes Universitários sobre a Realização de Atividades Extracurriculares na Graduação. Psicologia: Ciência e Profissão, 36(4), 864-876.

Pace, C. R. (1980). Measuring the quality of student effort. Current Issues in Higher Education, 1,10-19.

Pascarella, E., \& Terenzini, P.T. (2005). How college affects students: a third decada of research. (vol.2) San Francisco: Jossey-Bass.

Porto, R., \& Gonçalves, M. P.. (2017). Motivação e envolvimento acadêmico: um estudo com estudantes universitários. Psicologia Escolar e Educacional, 21(3), 515-522.

Quintas, H., Gonçalves, T., Ribeiro, C.M., Monteiro, R., Fragoso, A., Bago, J., Santos, L., \& Fonseca, H.M. (2014). Estudantes adultos no Ensino Superior: O que os motiva e o que os desafia no regresso à vida académica. Revista Portuguesa de Educação, 27(2), 33-56.

Ro, H.K., \& Knight, D.B. (2016). Gender differences in learning outcomes from the college experiences of engineering students. Journal of Engineering Education, 105(3), 478-507.

Shappie, A.T., \& Debb, S.M. (2017). African American student achievement and the historically Black University: the role of student engagement. Current Psychology, 1-13.

Soares, S. R., \& Cunha, M. I. (2017). Qualidade do ensino de graduação: concepções de docentes pesquisadores. Avaliação: Revista da Avaliação da Educação Superior, 22(2), 316-331.

Souza, V. C. (2017). Qualidade na educação superior: uma visão operacional do conceito. Avaliação: Revista da Avaliação da Educação Superior, 22(2), 332-357.

Vargas, H. M., \& Paula, M. de F. C. de (2013). A inclusão do estudante-trabalhador e do trabalhador-estudante na educação superior: desafio público a ser enfrentado. Avaliação, 18(2), 459-485.

Webber, K. L., Krylow, R. B., \& Zhang, Q. (2013). Does involvement really matter? Indicators of college student success and satisfaction. Journal of College Student Development, 54(6), 591-611.

Wolf-Wendel, L., Ward, K., \& Kinzie, J. (2009). A tangled web of terms: The overlap and unique contribution of involvement, engagement, and integration to understanding college student $\Lambda$ success. Journal of College Student Development, 50(4), 407-428.

Wu, C. (2011). Effects of college experiences on male and female student leadership capacity in Taiwan. Asia Pacifit Journal of Education, 12, 641-652.

Recebido 15/02/2018

$1^{a}$ Reformulação 01/07/2018

$2^{a}$ Reformulação 19/07/2018

Aceite Final 19/10/2018

Sobre as autoras

Camila Alves Fior é Psicóloga, Doutora em Educação pela UNICAMP, docente do departamento de Psicologia Educacional da Faculdade de Educação da UNICAMP. Membro do Grupo de Pesquisa: Psicologia e Educação Superior.

Elizabeth Mercuri é Psicóloga, Doutora em Educação pela UNICAMP, docente colaboradora da Faculdade de Educação da UNICAMP. Membro do Grupo de Pesquisa: Psicologia e Educação Superior. 